From the Buffon Needle Problem

to the Kreiss Matrix Theorem

\author{
Elias Wegert \\ Lloyd N. Trefethen
}

TR 92-1273

March 1992

Department of Computer Science Cornell University

Ithaca, NY 14853-7501 



\title{
From the Buffon Needle Problem to the Kreiss Matrix Theorem
}

\author{
Elias Wegert and Lloyd N. Trefethen
}

In this paper we present a theorem concerning the arc length on the Riemann sphere of the image of the unit circle under a rational function. But our larger purpose is to tell a story. We thought at first that the story began in 1962 with the Kreiss matrix theorem, the application that originally motivated us. However, our arc length question turns out to be more interesting than that. The story goes back to the famous "Buffon needle problem" of 1777 .

SPIJKER'S LEMMA IN THE COMPLEX PLANE. Let $r$ be a rational function of order $n$, that is, a quotient of two polynomials of degree at most $n$. Let $S$ denote the unit circle $\{z \in \mathbb{C}:|z|=1\}$, and let $\|\cdot\|_{1},\|\cdot\|_{2}$, and $\|\cdot\|_{\infty}$ denote the 1-, 2-, and $\infty$-norms on $S$,

$$
\|f\|_{1}=\int_{S}|f(z)||d z|, \quad\|f\|_{2}^{2}=\int_{S}|f(z)|^{2}|d z|, \quad\|f\|_{\infty}=\sup _{z \in S}|f(z)|
$$

Then the arc length of the curve $r(S)$ in the complex plane, which we denote by $L_{\mathbb{C}}(r(S))$, can be represented compactly by the formula

$$
L_{\mathbb{C}}(r(S)):=\left\|r^{\prime}\right\|_{1}
$$

If $r$ is multiplied by a constant $\alpha, L_{\mathbb{C}}(r(S))$ changes by the factor $|\alpha|$. However, this scale-dependence can be eliminated by considering the ratio

$$
L_{\mathbb{C}}(r(S)) /\|r\|_{\infty}
$$

Revised 3/18/92. To appear in the American Mathematical Monthly. 
In 1984, building on earlier work by Laptev, Strang, and Tadmor, LeVeque and Trefethen [9] observed that a bound on (1) could be used to derive a sharp form of the Kreiss matrix theorem (which we shall discuss at the end). They therefore posed the question, what is the maximum possible value of (1)?

It is easy to see that the value $2 \pi n$ can be attained; just take $r(z)$ to be $z^{n}$ or $z^{-n}$. If $r$ is restricted to be a polynomial, it follows from Bernstein's inequality that $2 \pi n$ is the maximum possible. It is also easy to see that $2 \pi n$ is the maximum value for rational functions in the special case $n=1$ (the reader can supply the proof!). Based on these facts and on computer experiments, it was conjectured in [9] that $2 \pi n$ is the maximum value (1) for all rational functions $r$ and all $n$. However, only the bound $4 \pi n$ was proved, and the task of eliminating this gap of a factor of 2 was presented as an Advanced Problem in this Monthly [10].

Just one response to the Monthly problem was received, from James C. Smith, of the University of South Alabama, who improved the bound to $2(2+\pi) n$ [16].

Five years later, Marc Spijker of the University of Leiden finally settled the conjecture in the affirmative [17]:

Theorem 1. $L_{\mathbb{C}}(r(S)) /\|r\|_{\infty} \leq 2 \pi n . \quad$ ("Spijker's lemma")

SPIJKER'S LEMMA ON THE RIEMANN SPHERE. The simplicity of Theorem 1 is marred by the need for the normalization by $\|r\|_{\infty}$. In looking for a cleaner formulation one may ask, what is the analogous result for the Riemann sphere? Let $\mathbb{S}$ denote the Riemann sphere $\left\{x \in \mathbb{R}^{3}:|x|=1\right\}$, with the north and south poles corresponding to the points $\infty$ and 0 in $\mathbb{C}$, respectively, according to the usual stereographic projection, and the equator corresponding to the unit circle $S$. This identification of $\mathbb{C}$ and $\mathbb{S}$ is discussed in many books on complex analysis [1], and it is readily shown that a unit of arc length $|d z|$ at a position $z \in \mathbb{C}$ is expanded by the factor $2 /\left(1+|z|^{2}\right)$ in being projected onto $\mathbb{S}$. It follows that if $r(S)$ is considered as a closed curve on $\mathbb{S}$, with $L_{\mathbb{S}}(r(S))$ denoting its arc length on $\mathbb{S}$, then we have

$$
L_{\mathbb{S}}(r(S)):=\left\|2 r^{\prime} /\left(1+|r|^{2}\right)\right\|_{1}
$$


Now, the trivial scale-dependence has been eliminated from the problem. It makes sense simply to ask, what is the maximum possible value of $L_{\mathbb{S}}(r(S))$ ?

The new result of this paper is the following answer to this question:

Theorem 2. $L_{\mathbb{S}}(r(S)) \leq 2 \pi n$. ("Spijker's lemma on the Riemann sphere")

The proof of Theorem 2 will emerge in the following pages. For the moment, we note first of all that like Theorem 1, Theorem 2 is obviously sharp, with equality attained for any $r$ that maps $S$ with winding number $n$ onto a great circle of $\mathbb{S}$. (For example, $r(z)=z^{n}$ maps $S$ with winding number $n$ onto the equator, and $r(z)=i^{n}(z-1)^{n} /(z+1)^{n}$ maps $S$ with winding number $n$ onto the Greenwich meridian.) A more important observation is that for any $r$ with $\|r\|_{\infty} \leq 1$, we have $L_{\mathbb{C}}(r(S)) \leq L_{\mathbb{S}}(r(S))$. This follows from (2), since $2 /\left(1+|r|^{2}\right) \geq 1$ when $|r| \leq 1$. Consequently, Theorem 2 implies Theorem 1 as a corollary. Thus Spijker's lemma on the Riemann sphere is both simpler and stronger than Spijker's lemma in the complex plane, and perhaps it should be considered the more fundamental result.

\section{FROM THE NEEDLE PROBLEM TO POINCARE''S FORMULA. The} reader has undoubtedly encountered the Buffon needle problem, published by the Comte de Buffon in 1777. Suppose a needle of length 1 is thrown at random on a plane ruled by parallel lines at a distance 1 apart. What is the probability that the needle will land in a position that crosses a line? Easy calculus shows that the answer is

$$
\text { Probability of intersection }=\frac{2}{\pi} \text {. }
$$

Buffon, incidentally, was the leading French naturalist of the eighteenth century and also a translator of Newton. He worked on his "problème de l'aiguille" long before publishing it as an appendix on "moral arithmetic" in his 44-volume treatise on natural history [3].

The needle problem became well known, especially among the French, and was generalized. Laplace, without referencing Buffon, solved the analogous problem for a square grid (Théorie Analytique des Probabilités, 1812). A more important generalization was 
to consider the slightly modified question: if the needle has length $L$, possibly greater than 1, what is the expected number of intersections? The answer is easily seen to be

$$
\text { Expected number of intersections }=\frac{2 L}{\pi} .
$$

And from here it is a small step mathematically, but a big one conceptually, to note that the same formula (3) is valid also for a paper clip. Various steps in this direction were taken by Cauchy, Lamé, and Barbier, among others [2]. In fact, if any rectifiable curve $\Gamma$ of arc length $L$ is thrown at random on the parallel grid, the expected number of intersections is (3). (A curve is rectifiable if its real and imaginary parts are functions of bounded variation [1].) The idea behind this result is that $\Gamma$ can be thought of as a concatenation of infinitesimal straight segments, each satisfying (3) for an appropriate infinitesimal value of $L$. Now it may seem at first that the expected number of intersections for $\Gamma$ should be more complicated than the sum of the expected numbers for the segments $\Gamma$ is composed of, since after all, the segments do not fall on the grid independently. However, independence is not relevant unless one cares about the efficiency of (3) as a method for approximating $\pi$. It is a basic fact of statistics that the expectation of a sum of random variables is equal to the sum of the expectations, regardless of whether or not they are independent. This observation seems elementary to us now, but its application to the needle problem was evidently not obvious in the nineteenth century.

Taking the paper clip to be a circle of radius $\frac{1}{2}$ gives an easy way to remember Buffon's result and its generalization (3). For this choice of $\Gamma, L$ is $\pi$ and the number of intersections is exactly 2 , no matter how the paper clip falls.

We now want to move from the plane to the sphere, a step taken as early as 1860 by Barbier [2]. Consider a "spherical paper clip"-that is, a curve $\Gamma$ embeddable in the Riemann sphere. Suppose $\Gamma$ is oriented at random on $\mathbb{S}$. What is the expected number of intersections with the equator? The answer is again essentially a matter of combining calculus with elementary statistics:

$$
\text { Expected number of intersections on the sphere }=\frac{L}{\pi} .
$$


Or one can skip the calculus and remember this result by thinking of the case in which $\Gamma$ is itself a great circle. In this case $L=2 \pi$ and the number of intersections is again exactly 2 unless $\Gamma$ happens to land exactly on the equator, an event of probability zero.

A final development completes this brief history. After Barbier, other mathematicians generalized these results further, including Poincaré, who referenced neither Buffon nor Barbier (Calcul des Probabilités, 1896 [12]). By this time it was clear that although the needle problem and its generalizations had conventionally been formulated as problems of probability, that interpretation could be dispensed with. Instead of orienting $\Gamma$ at random on $\mathbb{S}$ and asking for the expected number of intersections with a fixed equator, one can consider $\Gamma$ to be fixed on $\mathbb{S}$ and compute its arc length $L_{\mathbb{S}}(\Gamma)$ as an integral of the number of intersections with all great circles. To be precise, for any rectifiable curve $\Gamma \subseteq \mathbb{S}$ and any $x=\left(x_{1}, x_{2}, x_{3}\right) \in \mathbb{S}$, let $\nu(\Gamma, x)$ denote the number of points of intersection of $\Gamma$ with the great circle on $\mathbb{S}$ consisting of points equidistant from the antipodes $\pm x$. (When this number is infinite, the definition of $\nu(\Gamma, x)$ does not matter, for the set of such points has measure zero.) One obtains the following elegant result:

Lemma 1. $\quad L_{\mathbb{S}}(\Gamma)=\frac{1}{4} \int_{\mathbb{S}} \nu(\Gamma, x) d x . \quad$ ("Poincaré's formula")

The integral is taken with respect to area measure on $\mathbb{S}$.

Lemma 1 can be expressed in words as follows. To find the arc length of a curve on the Riemann sphere, integrate its numbers of intersections over all great circles, then divide by 4 . Or, equivalently, since the sphere has surface area $4 \pi$, take the average number of intersections and multiply by $\pi$. This latter paraphrase of Lemma 1 makes plain its equivalence to (4).

Poincaré's formula has far-reaching generalizations described in the book by Santaló [15], which the reader may consult for a wealth of related ideas as well as for the rigor lacking in the discussion above. It forms a centerpiece of the field known earlier as "geometric probability" but now as "integral geometry." 
PROOF OF SPIJKER'S LEMMA. Is it obvious now how to prove Theorem 2? All we need is the following lemma, whose proof we shall spell out though it might equally well have been left as an exercise. As above, $\nu(r(S), x)$ denotes the number of intersection points of the curve $r(S)$ with the great circle on the Riemann sphere $\mathbb{S}$ defined by the points $\pm x$.

Lemma 2. If $r$ is a rational function of order $n$, then $\nu(r(S), x) \leq 2 n$ for all $x \in \mathbb{S}$ with the possible exception of a single pair $x= \pm x_{0}, x_{0} \in \mathbb{S}$.

Proof. Since any point of $\mathbb{S}$ can be rotated to any other by a Möbius transformation, leaving the set of rational functions of order $n$ invariant, we are free to choose a particular value of $x$ for convenience. Let us take $x$ to be the north pole, so that the great circle in question is the equator, i.e., the image of $S$ on $\mathbb{S}$. If $r(z)=p(z) / q(z)$ for polynomials $p$ and $q$ of degree $\leq n$, then for $z \in S$,

$$
|r(z)|^{2}=\left|\frac{p(z)}{q(z)}\right|^{2}=\frac{p(z) \bar{p}(\bar{z})}{q(z) \bar{q}(\bar{z})}=\frac{p(z) p^{*}(z)}{q(z) q^{*}(z)}
$$

where $p^{*}(z):=z^{n} \bar{p}\left(z^{-1}\right)$ and $q^{*}(z):=z^{n} \bar{q}\left(z^{-1}\right)$. The condition $|r(z)|^{2}=1$ is thus a polynomial equation in $z$ of degree at most $2 n$. Therefore $r(S)$ intersects the equator in at most $2 n$ points, counted with multiplicity, unless it lies along the equator exactly. In the latter case it is obviously only the north and south poles for which the intersection number is infinite.

Since the surface area of $\mathbb{S}$ is $4 \pi$ and since $\frac{1}{4} \cdot 2 n \cdot 4 \pi=2 \pi n$, Theorem 2 is an immediate consequence of Lemmas 1 and 2 .

Spijker's original proof of Theorem 1, though derived independently, can be interpreted as a planar version of the same argument just given to establish Theorem 2. In particular, equation (6) of [17] is a kind of Poincaré formula for the complex plane, expressed in terms of lengths of one-dimensional projections instead of numbers of intersections. Apparently this formula was first worked out by Cauchy in 1832 and published by him in 1841 [5]. 
THE KREISS MATRIX THEOREM. What does all this have to do with the Kreiss matrix theorem? Let $A$ be an $n \times n$ matrix, and let $\|\cdot\|$ denote the matrix norm induced by the vector norm $\|\cdot\|_{2}$. The Kreiss matrix theorem, originally published in 1962 [8], concerns the the problem of characterizing matrices and families of matrices that are power-bounded. Let us define

$$
p(A)=\sup _{k \geq 0}\left\|A^{k}\right\|, \quad r(A)=\sup _{|z|>1}(|z|-1)\left\|(z I-A)^{-1}\right\| .
$$

The current, sharp form of the theorem reads as follows [17]:

Theorem 3. $r(A) \leq p(A) \leq \operatorname{en} r(A) . \quad$ ("Kreiss matrix theorem")

In words, a matrix $A$ is power-bounded $(p(A)<\infty)$ if and only if the norm of its resolvent $(z I-A)^{-1}$ increases at most inverse-linearly as $z$ approaches the unit circle from the outside $(r(A)<\infty)$. Moreover, the gap between $p(A)$ and $r(A)$ is a factor of at most $e(=2.718 \ldots)$ times $n$, so the same conclusion applies to families of matrices $\left\{A_{\nu}\right\}$ of fixed dimension that satisfy uniform bounds on $p\left(A_{\nu}\right)$ and $r\left(A_{\nu}\right)$.

The first inequality of Theorem 3 asserts that if $\left\|A^{k}\right\| \leq C$ for $k \geq 0$, then $\|(z I-$ $A)^{-1} \| \leq C /(|z|-1)$ for $|z|>1$. This is easy to prove by making use of the power series $(z I-A)^{-1}=z^{-1} I+z^{-2} A+z^{-3} A^{2}+\cdots$. The more interesting inequality is the second one, which asserts that if $\left\|(z I-A)^{-1}\right\| \leq C /(|z|-1)$ for $|z|>1$, then $\left\|A^{k}\right\| \leq e n C$ for $k \geq 0$. According to Tadmor's remarks in [18] for the earlier developments, the history of successive improvements toward this constant en involves no fewer than nine steps, though the earlier authors in the list were certainly not concerned with optimizing the constant:

$$
\begin{aligned}
\text { Kreiss '62: } & \sim[r(A)]^{n^{n}} \\
\text { Morton '64: } & \sim 6^{n}(n+4)^{5 n} \\
\text { Miller \& Strang '66: } & \sim n^{n} \\
\text { Miller '67: } & \sim e^{9 n^{2}}
\end{aligned}
$$




$$
\begin{aligned}
\text { Laptev '75 / Strang '78: } & 32 e n^{2} / \pi \\
\text { Tadmor '81: } & 32 e n / \pi \\
\text { LeVeque \& Trefethen '84: } & 2 e n \\
\text { Smith '85: } & e\left(1+\frac{2}{\pi}\right) n \\
\text { Spijker '91: } & e n
\end{aligned}
$$

History, thank goodness, stops here. It is shown in [9] that the constant en is best possible.

As the estimates have become sharper, the proofs have become mercifully simpler and have ceased to depend upon the explicit manipulation of eigenvalues and normal forms of matrices. We reproduce now the argument from [9] that shows how the constant en follows from Spijker's lemma.

Proof of the second inequality of Theorem 3. According to the calculus of resolvents described for example in [7], the matrix $A^{k}$ can be written as the Cauchy integral

$$
A^{k}=\frac{1}{2 \pi i} \int_{G} z^{k}(z I-A)^{-1} d z
$$

where $G$ is any curve enclosing the eigenvalues of $A$, which must lie in $\{z \in \mathbb{C}:|z| \leq 1\}$ if $r(A)<\infty$. Let $u$ and $v$ be arbitrary $n$-vectors with $\|u\|_{2}=\|v\|_{2}=1$. Then

$$
v^{*} A^{k} u=\frac{1}{2 \pi i} \int_{G} z^{k} q(z) d z,
$$

where $v^{*}$ denotes the conjugate transpose of $v$ and $q(z)$ is the function $v^{*}(z I-A)^{-1} u$, which can be shown to be a rational function of order $n$. Integration by parts gives

$$
v^{*} A^{k} u=\frac{-1}{2 \pi i(k+1)} \int_{G} z^{k+1} q^{\prime}(z) d z .
$$

Let the contour of integration be taken as $G=\left\{z \in \mathbb{C}:|z|=1+(k+1)^{-1}\right\}$. On this contour we have $\left|z^{k+1}\right| \leq e$ and hence

$$
\left|v^{*} A^{k} u\right| \leq \frac{e}{2 \pi(k+1)} \int_{G}\left|q^{\prime}(z)\right||d z| .
$$


This integral can be interpreted as the arc length of $q$ over the circle $G$. By a trivial change of variables it might as well be an arc length over the unit circle $S$. Theorem 1 therefore implies

$$
\left|v^{*} A^{k} u\right| \leq \frac{e}{2 \pi(k+1)}(2 \pi n) \sup _{z \in G}|q(z)|
$$

and therefore, since the supremum of $|q(z)|$ on $G$ is at most $(k+1) r(A)$, by the definition of $r(A)$, we have

$$
\left|v^{*} A^{k} u\right| \leq \operatorname{enr}(A) .
$$

Finally, we note that since $\left\|A^{k}\right\|$ is the supremum of $\left|v^{*} A^{k} u\right|$ over all vectors $u$ and $v$ with $\|u\|_{2}=\|v\|_{2}=1$, this last inequality proves the theorem.

The Kreiss matrix theorem has been a fixture of numerical analysis since its appearance in 1962 and dissemination in the well-known book by Richtmyer and Morton [14]. It is one of the fundamental results available for establishing numerical stability of discrete processes.

CONCLUSION. From Buffon to Spijker to Kreiss, the pieces of our story fit together so neatly that it may seem there can be nothing more to say. Nevertheless, matters related to the Kreiss matrix theorem are subjects of active interest today, and in conclusion, we would like to mention a recent generalization of Theorem 3 and an open question.

The generalization concerns the problem of numerical stability of the "method of lines." When time-dependent partial differential equations are solved numerically by discretization, it is common to simplify the process by constructing the space discretization and the time discretization independently. For example, the Crank-Nicolson formula for solving parabolic PDEs, of which the prototype is the heat equation $u_{t}=u_{x x}$, can be viewed as a second-order centered finite difference with respect to $x$ coupled with the "trapezoid formula" with respect to $t$. In more realistic problems the space discretization might involve more complicated finite difference, finite element, or spectral approximations and the time discretization might be accomplished by any of the familiar methods for ODEs such as Runge-Kutta or Adams-Bashforth formulas [6]. 
According to the celebrated Lax Equivalence Theorem, the numerical solution computed by a consistent discretization of a well-posed linear partial differential equation will converge to the solution of the PDE as the mesh size shrinks to 0 if and only if the discretization is numerically stable [14]. (We ignore the effects of rounding errors.) But how does one test for numerical stability? It has recently been shown that for method of lines calculations, one can do it by a transplantation of the Kreiss matrix theorem from the unit disk to the subset of $\mathbb{C}$ known as the stability region of the ODE formula [13]. One replaces the monomial $A^{k}$ in the term $p(A)$ of Theorem 3 by the solution to a more general matrix recurrence relation, and the unit disk in the term $r(A)$ of Theorem 3 by the stability region. The condition for stability is that the norm of the resolvent of an appropriate spatial discretization matrix must increase at most inverse-linearly as $z$ approaches the boundary of the stability region from the outside. For numerical analysts, to whom stability regions of ODE formulas are as familiar as simple groups are to algebraists, this result provides an easy means of applying the Kreiss matrix theorem to a wide range of practical problems. In particular it is applicable to the stability analysis of the high-accuracy numerical techniques known as spectral methods [4], where the matrices that arise are often far from normal and difficult to analyze by more elementary techniques.

The open question is, what happens to Theorem 3 if $r(A)$ is viewed as a constant rather than a variable? If $r(A)=1$, then it can be shown that the field of values of $A$, that is, the set of Rayleigh quotients $u^{*} A u /\|u\|_{2}^{2}$, must lie in the closed unit disk. By a result due originally to Lax and Wendroff and subsequently sharpened by Halmos, Berger, and Pearcy [14], it follows that when $r(A)=1$ we have $p(A) \leq 2$, or in other words, the factor $e n$ of Theorem 3 can be replaced by the constant 2, independently of $n$. Now, what if $r(A)$ is a constant greater than 1 ? For example, what can be said about $p(A)$ if $r(A)=2$ ? It is known that $p(A)$ can no longer be bounded by a constant [11], but beyond this-for example, whether en can be improved to a quantity that grows only logarithmically in $n$-nothing is known. 
ACKNOWLEDGMENTS. The problem of generalizing Spijker's lemma to the Riemann sphere was raised by the second author at a meeting in Oberwolfach in February, 1991. The proof presented here was devised by the first author, who is grateful to D. Stoyan for pointing out the connections with integral geometry. Subsequently A. I. Aptekarev of the Keldysh Institute of Applied Mathematics in Moscow has communicated to us a different and equally simple proof based on induction in $n$.

We are grateful also for advice from a number of others, especially Marc Spijker.

This paper was written during a visit by the second author to the Université Pierre et Marie Curie in Paris, across the street from the Jardin des Plantes where Buffon served as Director 250 years ago and his statue stands today.

\section{REFERENCES}

1. L. Ahlfors, Complex Analysis, McGraw-Hill, 1966.

2. E. Barbier, Note sur le problème de l'aiguille et le jeu du joint couvert, J. Math. Pures Appl., 5 (1860) 273-286.

3. G. Buffon, Essai d'arithmétique morale, Supplément à l'Histoire Naturelle, v. 4, 1777.

4. C. Canuto, M. Y. Hussaini, A. Quarteroni, and T. A. Zang, Spectral Methods in Fluid Dynamics, Springer-Verlag, 1988.

5. A. Cauchy, Note sur divers théorèmes relatifs à la rectification des courbes, et à la quadrature des surfaces, Comptes Rendus, 13 (1841), p. 1060, reprinted in A. Cauchy, Oeuvres, Serie I, v. 6, pp. 369-375, Gauthier-Villars, Paris, 1888.

6. E. Hairer, S. P. Nørsett, and G. Wanner, Solving Ordinary Differential Equations, Springer-Verlag, 1987 (v. 1) and 1991 (v. 2).

7. T. Kato, Perturbation Theory for Linear Operators, 2nd ed., Springer-Verlag, 1976.

8. H. O. Kreiss, Über die Stabilitätsdefinition für Differenzengleichungen die partielle Differenzialgleichungen approximieren, BIT, 2 (1962) 153-181.

9. R. J. LeVeque and L. N. Trefethen, On the resolvent condition in the Kreiss Matrix Theorem, BIT, 24 (1984) 584-591.

10. R. J. LeVeque and L. N. Trefethen, Problem \#6462, Advanced Problems, Amer. Math. Monthly, 91 (1984) 371.

11. C. A. McCarthy and J. Schwartz, On the norm of a finite Boolean algebra of projections, and applications to the theorems of Kreiss and Morton, Comm. Pure Appl. Math., 18 (1965) 191-201.

12. H. Poincaré, Calcul des Probabilités, Gauthier-Villars, Paris, 1896.

13. S. C. Reddy and L. N. Trefethen, Stability of the method of lines, Numer. Math., to appear.

14. R. D. Richtmyer and K. W. Morton, Difference Methods for Initial-Value Problems, 2nd ed., WileyInterscience, 1967. 
15. L. A. Santaló, Integral Geometry and Geometric Probability, Addison-Wesley, 1976.

16. J. C. Smith, An inequality for rational functions, Solutions of Advanced Problems, Amer. Math. Monthly, 92 (1985) 740-741.

17. M. N. Spijker, On a conjecture by LeVeque and Trefethen related to the Kreiss matrix theorem, $B I T, 31$ (1991) 551-555.

18. E. Tadmor, The equivalence of $L_{2}$-stability, the resolvent condition, and strict $H$-stability, Lin. Alg. Applics., 41 (1981) 151-159.

Fachbereich Mathematik

Bergakademie Freiberg

D-O-9200 Freiberg

Germany

wegert@mathe.ba-freiberg.dbp.de
Department of Computer Science

Cornell University

Ithaca, $N Y 14853$

USA

lnt@cs.cornell.edu 